\title{
EL CLÚSTER COMO MODELO DE RED EMPRESARIAL, UNA OPCIÓN RELEVANTE PARA LA COMPETITIVIDAD DEL SUBSECTOR DEL TURISMO EN COLOMBIA
}

\author{
THE CLUSTER AS MODEL OF NETWORK BUSINESS, A RELEVANT OPTION \\ FOR THE COMPETITIVENESS OF THE COLOMBIA TOURISM SUBSECTOR
}

\section{O CLUSTER COMO MODELO DA REDE DE NEGÓCIOS, UMA OPÇÃO RELEVANTE PARA A COMPETITIVIDADE DO SECTOR SECUNDÁRIO DO TURISMO NO DEPARTAMENTO DE CUNDINAMARCA (COLÔMBIA)}

Por: GIL TOLEDO - John Jairo

Doctorante en Administración, Universidad de Celaya, México. Docente Asociado II, Universidad Jorge Tadeo Lozano. Email: johnj.giltoledo@utadeo.edu.co, Colombia.

\section{RESUMEN}

El escenario empresarial existente en este tiempo le propone a las compañías el reto de coexistir en el ambiente de sistemas organizacionales asociados, específicamente para las micro, pequeñas y medianas empresas, y su aporte al crecimiento de la región. Esta situación ha despertado inquietud en los investigadores por analizar este tejido empresarial a partir de su impacto en la ejecución de las firmas. A partir del anterior raciocinio, este artículo analiza el impacto que genera el vínculo existente entre empresas y el establecimiento en relación a los procesos de creatividad empresarial en los municipios del departamento de Cundinamarca (Colombia). El presente documento es empírico y los resultados obtenidos forman parte de la construcción del marco teórico que da sustento a la investigación que la genera. El producto de este trabajo muestra que los lazos que vinculan a las organizaciones empresariales analizadas con el fin de llevar a cabo acciones de Investigación y Desarrollo y transmisión de conocimientos, contribuyen de manera provechosa al crecimiento de las organizaciones.

Este trabajo forma parte de una revisión bibliográfica de investigación que posterioremente se consolidará como una propuesta de mayor impacto y relevancia para el sector objeto de estudio.

Palabras clave: Clúster, empresarial, competitividad.

JEL: M10, L10, R58 


\section{ABSTRACT}

The business scenario existing at this time development proposes to companies challenge coexist associated, specifically for the micro, small and medium enterprises in the environment of organizational systems, and their contribution to the growth of the region. This situation has aroused concerns in researchers to analyze this business from its impact on the execution of the signatures. From the above reasoning, this article analyzes the impact generated by the link between companies and the establishment in relation to processes of entrepreneurial creativity in the municipalities of the Department of Cundinamarca (Colombia). This document is empirical, and the results are part of the construction of the theoretical framework that gives support to research that generates it. The product of this work demonstrates that ties linking business organizations analyzed in order to carry out research and development and transmission of knowledge, helpful way contribute to the growth of organizations.

This work is part of a literature review of research action be strengthened as a proposal for greater impact and relevance to the sector the subject of study.

Keywords: Cluster, business, competitiveness.

JEL: M10, L10, R58

\section{RESUMO}

O cenário de negócios existentes neste momento desenvolvimento propõe desafio as empresas coexistem associado, especificamente para as micro, pequenas e médias empresas no ambiente de sistemas organizacionais e sua contribuição para o crescimento da região. Esta situação suscitou preocupações em pesquisadores para analisar este negócio de seu impacto sobre a execução das assinaturas. O raciocínio acima, este artigo analisa o impacto gerado pela relação entre as empresas e o estabelecimento em relação aos processos de criatividade empreendedora nos municípios do departamento de Cundinamarca (Colômbia). Este documento é empírico, e os resultados são parte da construção do quadro teórico que dá suporte à pesquisa que gera-lo. O produto deste trabalho mostra que os laços que vinculada ao negócio as organizações analisado para executar ações de pesquisa, desenvolvimento e transmissão de conhecimento, contribuir de maneira de maneira útil para o crescimento das organizações. Este trabalho fazem parte de uma revisão bibliográfica de pesquisa que pinos consolida-se como uma proposta de maior impacto e relevância para o objeto do setor de estudo.

Palavras chave: Cluster, negócios, competitividade.

JEL: M10, L10, R58 


\section{INTRODUCCIÓN}

La contribución del turismo al PIB nacional colombiano se ha analizado con fundamento en los resultados económicos de la actividad a la luz de la hipótesis de que la contribución al PIB y la creación de puestos de trabajo, contribuyen a la concepción de resultados provechosos en la riqueza regional. La diversidad de situaciones presentes en las distintas formas de crecimiento turístico a examinar en el Departamento de Cundinamarca, permiten evidenciar la utilidad de excavar en la correspondencia entre clúster turístico y crecimiento, diferenciando entre competitividad real y competitividad potencial. Del estudio de las evidencias empíricas y de la teoría existente al respecto surgen varias preguntas detonadoras que dan origen al proyecto de investigación. ¿Cuáles son y en qué escenario actual se encuentran las empresas de la cadena productiva del turismo del departamento de Cundinamarca, para conformar un clúster? ¿Cuál sería la estructura ideal para un clúster de este tipo de empresas? ¿Qué políticas y normas se pueden manifestar y plantear para la concepción de un clúster que aglutine estas empresas? De igual manera, y con fundamento en la riqueza conceptual usada para revisar la organización productiva regional y los elementos componentes de la competitividad en el departamento, asoma una pregunta con respecto del beneficio del concepto de clúster para estudiar los rumbos turísticos que son materia de análisis en este estudio. Con el fin de contestar estas inquietudes planteadas, se toma como elemento de inicio el fundamento teórico de desarrollo empresarial en el departamento y se plantea un esquema de observación sistémica de los protagonistas que actúan en la actividad turística. Este esquema contribuye con un ambiente general para el estudio de la noción de clúster turístico y el estudio de los elementos importantes.

El ambiente influencia en la conducta de la organización y recíprocamente (Minguzzi y Passaro, 2000), es por esto que la empresa requiere apropiarse de su estado de progreso, como también el de la industria con el propósito de incrementar su competencia de adiestramiento (Benson-Rea y Wilson, 2003), de igual manera, las relaciones con otros protagonistas que confluyen en el panorama regional, convirtiéndose en la vía para la consolidación de ventajas competitivas organizacionales y departamentales (Carbonara, 2002; Feldman, Francis y Bercovitz, 2005).

\section{REVISIÓN DE LA LITERATURA}

Según Porter (1998), el concepto de clúster se utiliza para analizar la aglomeración de empresas e instituciones, que en un espacio geográfico determinado se relacionan en base a la producción de bienes y servicios. En la actividad turística, se ha generalizado este concepto debido a que resulta muy útil para analizar el conjunto de empresas que prestan servicios en torno a los atractivos turísticos y la relación de estas con las instituciones del sector público y privado que integran la superestructura. 
Las diferentes definiciones halladas en literaturas en las cuales se han abarcado puntualmente estos conceptos:

1. Destino como una zona, área, sitio o lugar geográfico delimitado (Lanquar, 2001).

2. Destino como centro de producción turística, o sea, como la confluencia entre oferta y demanda o espacio donde se produce la experiencia turística (Manente y Minghetti, 2006).

3. Destino como sistema, clúster, polo de desarrollo o red de proveedores en función de la actividad turística (Lanquar, 2001).

4. Destino como imagen percibida o creada por viajeros, expertos en turismo y medios de comunicación (Stabler, 1988; Telisman-Kosuta, 1989; Gallarza et al., 2002).

Para poder lograr una visión integradora del proceso de gestión del destino turístico como unidad analítica esencial del turismo e introducirse en el análisis de los modelos que lo intentan explicar, es imprescindible analizar el fenómeno turístico de manera más general, a partir de los modelos que describen su estructura funcional según los postulados de la Teoría General de Sistemas de Von Bertalanffy ${ }^{1}$

\subsection{Modelos estructurales-Funcionales}

2.1.1 Entre los modelos básicos se encuentra el de Leiper de 1979, adaptado en 1990 y posteriormente asumido como referente teórico a partir de esa década, pues "logró centrar la atención en el concepto de flujo turístico (viajeros de ida y viajeros de regreso)" (Martín, 2006: 19). Explica el turismo como una relación de intercambio entre regiones generadoras (emisoras) de viajeros y regiones de destino turístico (receptoras), mediante regiones de tránsito en ruta donde se ubican los componentes de la industria turística.

2.1.2 Poco antes Miossec (1977) presentó las interacciones de cuatro elementos en el proceso de desarrollo de un destino:

- $\quad$ El destino en sí mismo y sus características.

- $\quad$ El papel del transporte.

- Patrones de conducta de los turistas.

- Actitudes de los tomadores de decisiones y de los residentes en el destino.

1. Karl Ludwig von Bertalanffy fue un biólogo y filósofo austríaco, reconocido fundamentalmente por su teoría de sistemas. 


\subsection{Modelos teóricos de destinos turísticos orientados a la competitividad}

Los modelos que aquí se abordan no tienen una denominación explícita como modelos de gestión, sin embargo, en casi todos se pone de manifiesto la noción de sistema de los destinos y son recurrentes las variables "competitividad" y "sostenibilidad", en una relación biunívoca o más que todo interdependiente.

2.2.1 Modelo Pentagonal del Sistema Turístico (Martín, 2006), que intenta resolver las insuficiencias de los modelos anteriores basándose en una concepción multisistémica del turismo, al hacer grandes subdivisiones del mismo en: subsistema endógeno o propiamente turístico, subsistema exógeno o entorno turístico y macroentorno o ambiente general. Los elementos de los subsistemas endógeno y exógeno y sus enlaces conforman el "contenido" del fenómeno turístico, inserto en un proceso de interrelaciones con el macroentorno al que denomina "dinámica turística", "operación turística" o "proceso del turismo", el cual conduce a unos resultados o "impactos" (Martín, 2006: 23).

\subsection{Modelo Conceptual de Competitividad y Sostenibilidad para Destinos Turísticos}

Desarrollado por Ritchie y Crouch en 2000 y perfeccionado en 2003, el cual se considera uno de los modelos teóricos más influyentes de los últimos años. Conocido como Modelo de Calgary, son recurrentes en el mismo las ideas sobre el sistema turístico como un sistema abierto, el enfoque de marketing en el análisis del entorno competitivo (en realidad no dista mucho del socorrido análisis del modelo de las cinco fuerzas de Porter) y la transversalidad de la actividad turística en la interconexión de distintas cadenas de valor.

\subsection{Cluster Turístico}

El turismo es uno de los tres principales sectores económicos de la actualidad. Es por esto mismo, que según lo que nos explica Porter (1998), quien afirma que "en la actividad empresarial actual se manifiesta el fenómeno en que algunas fuentes de ventajas comparativas tradicionales quedan anuladas, o al menos sus efectos son menores, por la facilidad de accesos que la globalización permite”, significa que en muchos países están buscando nuevas formas de atraer a los turistas, puesto que algunos métodos ya han quedado obsoletos, las demandas de los usuarios cada día son mayores, ahora cada día más todo es más exigente, se requiere una renovación, acorde al mundo globalizado en que vivimos, la alta competitividad en la globalización ha formado una nueva configuración empresarial denominada Clúster. Además, cabe señalar, que se están aprovechando y estudiando los resultados concretos de clústeres turísticos en países como Estados Unidos, Canadá, Australia, México, el Caribe y Costa Rica, que han alcanzado posiciones exitosas sostenibles en el mercado. En turismo, los clústeres comprenden agrupamientos de varios atractivos turísticos concentrados en una región geográfica, con infraestructura compatible, equipamientos, servicios receptivos y órganos y agentes turísticos coordinados para ofrecer un producto turístico integrado y diferenciado. Éstos 
complejos turísticos son los que más han traído la atención de los inversionistas, es una de las aéreas que más crece en el mercado.

\subsection{Tendencias teóricas sobre agrupamientos geográficos, clústeres regionales y redes}

A partir de que 1990 Michael Porter sacara a la luz pública su libro La ventaja competitiva de las naciones, el estudio de los clúster ha crecido de manera sustancial. A esto contribuyó de una parte la influencia a partir de los años 80, de un conjunto de tendencias económicas, tales como:

- Las nuevas teorías sobre la innovación, a la luz de la teoría general de sistemas dentro de los conceptos de geografía económica (fundamentada en la estructura neomarshalliana de los distritos industriales).

- Nuevas teorías del crecimiento económico y del comercio exterior

- Fundamentos teóricos como la teoría de los costes de transacción, de la teoría de la firma y de la información teórica fundamentada en los recursos.

Estas tendencias se fundamentan en los cambios del ambiente local de conocimiento en el que se desenvuelven las organizaciones y que ha sido analizada a profundidad por varios expertos que entre otros análisis, se dedicaron al estudio de escenarios regionales en el norte italiano, regiones danesas y en el estado Germano de Baden-Württemberg, entre otras, estudios que tienen elementos comunes en la profundidad de sus raíces culturales, históricas, sus instituciones y en desarrollos económicos regionales que dan fundamento a estas aglomeraciones.

Evocando los conceptos de Porter (1998), un modelo asociativo como el clúster puede afectar el desarrollo competitivo en tres escenarios a saber:

1.- Incrementa el proceso productivo empresarial de los miembros del clúster.

2.- Favorece la capacidad de innovación y derivado de lo anterior, se incrementa la productividad de las empresas involucradas y

3.- Promueve la creación de nuevas empresas, lo que fortalece la innovación y el crecimiento del clúster.

Derivado de las conversaciones preliminares sostenidas con los empresarios, previo a la presentación del presente proyecto, se colige también que los empresarios se preocupan por desarrollar competencias y ventajas que les permitan compartir experiencias y colaborar con sus pares, para no solo consolidar sus posiciones en el escenario empresarial del departamento, sino estar siempre adelante en la oferta de servicios.

En la siguiente gráfica se evidencian las diferentes teorías que han estudiado los clústeres desde el concepto de la teoría económica, como aporte al desarrollo de los modelos empresariales. 
Figura 1. Modelos teóricos de Clústeres Industriales

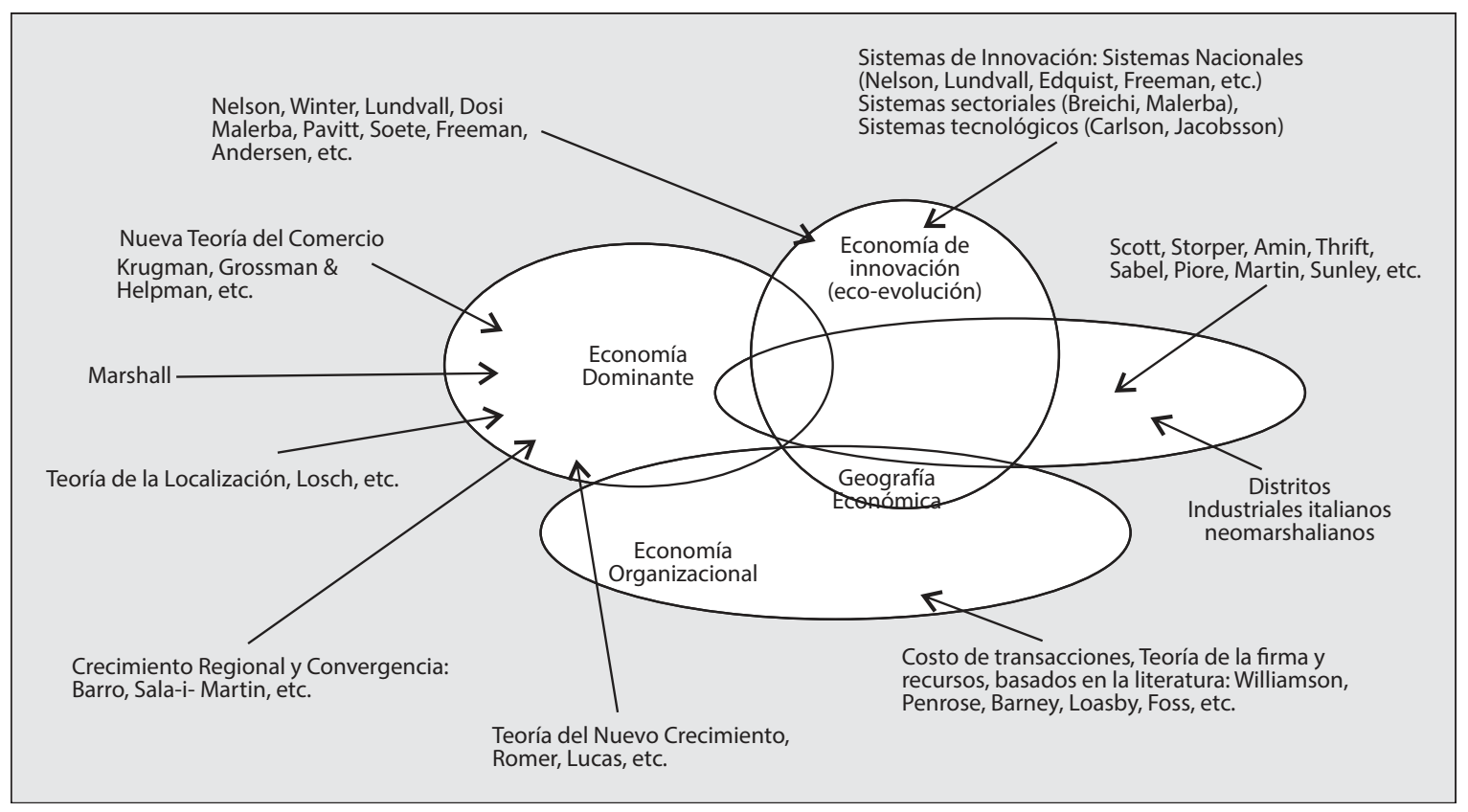

Fuente: elaboración propia a partir de Dahl. (2001)

Se fundamentará el marco conceptual de este artículo, además de la revisión teórica existente, en la Teoría de Clústeres Industriales de Michael Porter (1998) y en el modelo de Integrado de Competitividad de Dwyer y Kim (2003).

Este último modelo se soporta en 3 conjuntos de componentes: Un componente de recursos, un componente del entorno circundante, y un componente centrado en la competitividad del destino turístico objeto de análisis. En la figura 2 se describe el modelo

Figura 2. Modelo Integrado de competitividad de Dwyer y Kim

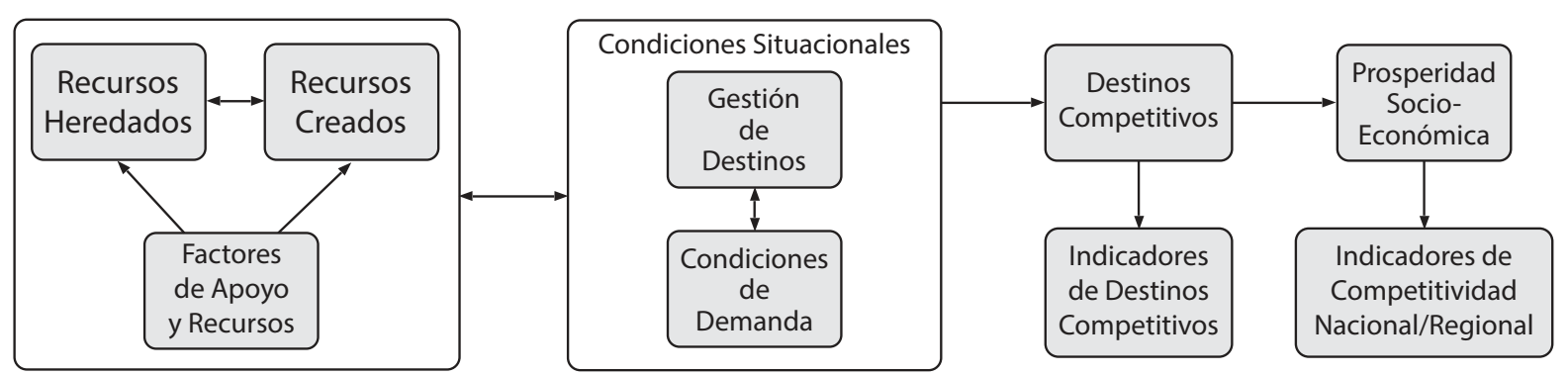

Fuente: Dwyer y Kim (2003)

Los recursos analizados en el modelo se agrupan en dos tipos: Los recursos fundamentales concebidos (naturales o culturales) o heredados (eventos especiales, 
tiendas, alojamiento, etc.), y los recursos de soporte que se relacionan con los mecanismos de acceso, la atención y el servicio, el alojamiento y vínculos del mercado.

- Los requisitos situacionales corresponden a las fuerzas del entorno que afectan positiva o negativamente los resultados en materia de competitividad del destino. Hacen referencia tanto a las variables del micro como al macro entorno, e involucran elementos de planeación y crecimiento, marketing, gestión humana y manejo ambiental.

- $\quad$ El tercer bloque de elementos se orienta a la competitividad del destino turístico analizado y que es resultado de la integración de los grupos de elementos mencionados en los párrafos anteriores y se relaciona con el crecimiento del departamento y del país y el crecimiento y la mejora en las condiciones de vida de los vecinos del sector.

\subsection{Las teorías de la ubicación y de la geografía económica}

Krugman (1991) tomó los conceptos de Marshall (1920), e implementó la noción de ventajas de aglomeración asociadas a los resultados crecientes a escala. De igual manera, pudo identificar tres elementos que consolidan la concentración de una tarea en un lugar específico:

- Primeramente. Se establece un centro industrial, que favorece empresas como a empleados.

- Un centro industrial facilita el abastecimiento, aprovechando las economías de escala.

- De igual manera, un centro industrial da como resultado lo que Krugman (1992) llama ósmosis tecnológica (technological spillovers).

El fundamento de estos escenarios teóricos hace énfasis en la importancia del peso porcentual del costo de transporte en la estructura final del costo, por eso, algunas tareas dentro de la cadena productiva se establecen prioritariamente próximos a los centros de abastecimiento de materia prima, mientras que otras se ubican cerca de los espacios de comercialización, al mismo tiempo que otras se establecen a la mitad del camino o en otro espacio geográfico. La clave más importante en este tipo de modelos geográficos es la distancia, o específicamente, el costo en términos de tiempo y recursos de las largas distancias entre procesos.

Para Krugman, la concentración es la prueba de que existen rendimientos crecientes en las economías de los países. Krugman en su libro Geography And Trade, propone dos interrogantes: (a) qué motivos o razones permitieron la agrupación entre los productores; y (b) por qué este agrupamiento se sucedió en primera instancia. La primera inquietud tiene su respuesta en un modelo planteado por el autor, que se fundamenta en la interacción de los rendimientos crecientes, la demanda y los costos de transporte de los bienes. Con el fin de satisfacer la demanda local, un productor intentará estar lo más cerca posible de sus proveedores, con el propósito de reducir los costos de desplazamiento de sus productos. Este círculo productivo se intenta mantener y retroalimentar permitiendo la aglomeración de 
empresas y con ello el logro de economías de escala en los costos de transporte. No obstante lo que pueda parecer este modelo, el autor parece aceptar de soslayo un ciclo de vida de este tipo de concentraciones geográficas de empresas.

Con el objetivo de fundamentar sus investigaciones, Krugman (1991) nos muestra evidencia fundamentada en datos, a partir del análisis de las concentraciones geográficas en Estados Unidos utilizando el coeficiente de Gini en distintas industrias, evidenció el porcentaje de participación del empleo en la industria americana, como el porcentaje de contribución al empleo en manufactura nacional, en la industria específica objeto de análisis. A partir de la aplicación de estos indicadores, se pudo concluir, que en los Estados Unidos, existe alta concentración industrial Krugman (1991).

\subsection{Modelo auto-organizativo de clústeres industriales}

Este modelo intenta explicar las deficiencias planteadas en el modelo de Krugman y parte de la aceptación de que los clústeres industriales son conglomerados de empresas con estructuras. Buendía (2005) propone el análisis de este modelo con fundamento en la mecánica de sistemas, a partir del entendimiento de la interacción de un número mayor de variables al analizado por Krugman en este tipo de agrupaciones industriales. A continuación se presentan las variables analizadas por Buendía en su propuesta.

- El crecimiento de la economía

- Un desarrollo local en materia de infraestructura

- La ventaja competitiva aunada a la posición en materia de exportaciones

- El crecimiento propio del clúster

- El número de empresas localizadas en el espacio geográfico

- El tamaño mismo de cada empresa miembro del clúster

- La oferta de mano de obra calificada

- La disponibilidad de recursos, materia prima e insumos

- Los procesos productivos

- La innovación

- El conocimiento acumulado

- La cantidad de recursos dispuestos para I+D

- Las utilidades derivadas del ejercicio empresarial

- Los indicadores de competitividad

- Los factores que afectan el correcto funcionamiento de una economía de aglomeración

- Demanda local saturada

- Costo excesivo de los terrenos donde se ubican los agrupamientos

- Costos altos de infraestructura, con escasez de la misma. 
Este modelo fundamenta su análisis en las variables estudiadas por Porter (1990) y por Krugman (1991), como los detonadores de la competitividad de los clústeres. Buendía (2005) fundamenta su análisis en el nivel de complejidad de los clústeres industriales y los niveles de retroalimentación de los mismos, por lo que usa la dinámica de sistemas para observar su comportamiento e intentar la formalización del modelo propuesto, ayudado por la teoría de las urnas.

Figura 3. Modelo Auto-organizativo de Clústeres Industriales

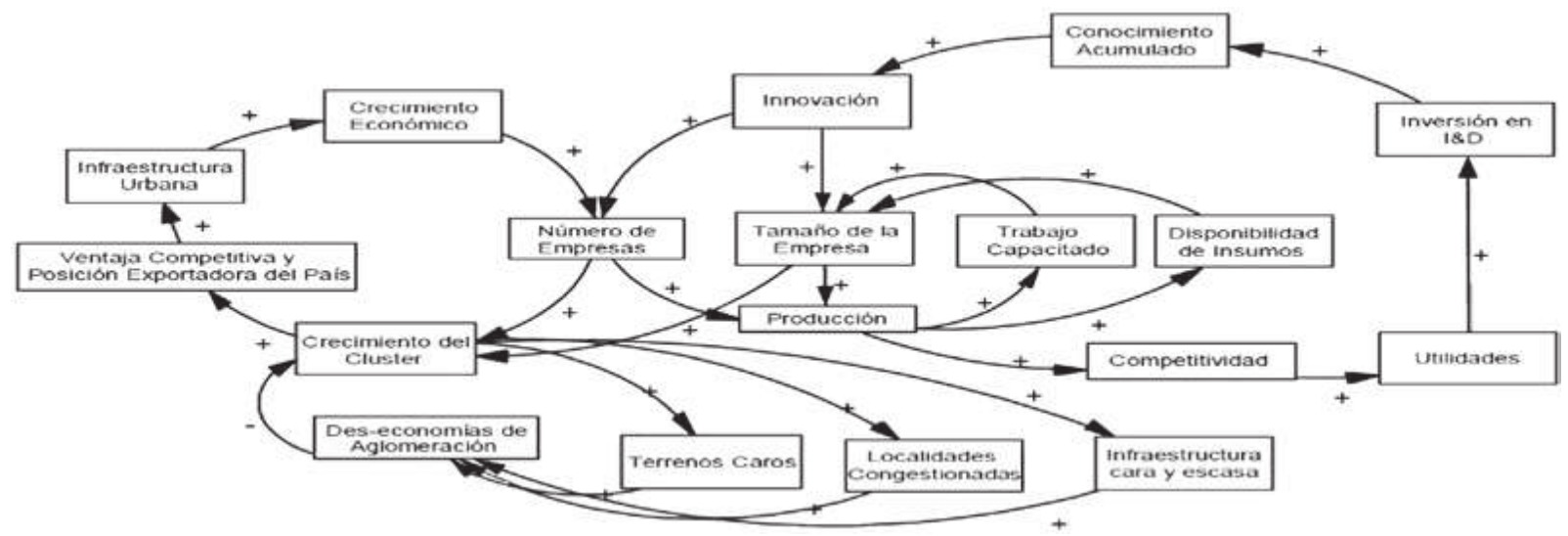

Fuente: Buendía (2005).

\subsection{La teoría de la interacción y "los distritos industriales"}

Las uniones e interacciones traen como resultado repeticiones que consolidan las relaciones, fundamentadas en la certidumbre y credulidad y minimizan los costos de los procesos y su administración. Igualmente, la interacción impulsa la innovación, edificando lo que es un bien para la sociedad apropiado por las empresas que conforman el distrito. Por esta razón, una interacción frecuente en el plano local o regional se evidencia en desarrollo de tecnología, y ahorro por economías de escala para el bienestar de todos los miembros del distrito.

Marshall (1920) define un distrito industrial como:

... una concentración, en un área geográficamente limitada, de empresas... especializadas en un sector dominante. El elemento clave de modelo de organización es el factor unificador; es decir, aquel que permite la integración racional y organizada de los numerosos sujetos que componen el distrito y al que se denomina "atmósfera industrial", entre sus elementos se destacan: la cultura productiva, el conocimiento de los demás sujetos que forman parte de la comunidad y los vínculos existentes con éstos, y las tradiciones histórico-políticas comunes. Esta atmósfera es el verdadero canal de comunicación entre los diferentes sujetos. Es el factor que hace posible la manifestación y la difusión de un hábito de colaboración e intercambio de información y de experiencias aun en un contexto altamente competitivo. 


\subsection{La teoría de los Clústeres industriales}

Las cifras actuales de crecimiento y desarrollo, cuya característica fundamental es una gran incoherencia entre lo global y lo nacional o regional, en donde estos últimos se constituyen en actores fundamentales en el nuevo contexto orbital, de esta realidad se deriva un nuevo contexto dentro del panorama productivo a través de los procesos de unión o agrupación, los cuales popularizó Porter (1990). Estas aportaciones acuñaron el término complejos productivos, tan en boca hoy en día en la nueva realidad empresarial del mundo. Estos complejos contribuyen sustancialmente en el panorama productivo de las organizaciones. Abandonando temporalmente el concepto de clúster, debemos retomar el análisis del turismo como una de las manifestaciones más enérgicas y crecientes de la sociedad actual. Aunque las actividades de viajes han estado presentes en todas las etapas del desarrollo de la humanidad, fueron las condiciones de vida de la sociedad del siglo XX (aumento del tiempo libre, conquista masiva de derecho a las vacaciones, disponibilidad de ingreso, mejoras tecnológicas en transporte y comunicaciones) las que definitivamente permitieron su rápida evolución.

La Organización Mundial del Turismo ha podido determinar que el ecoturismo en asocio con el turismo de aventura, el agroturismo, el turismo de cruceros y el turismo cultural demandará la mayor atención de los consumidores de este tipo de servicios. El ecoturismo, en consecuencia con este comportamiento, ha adquirido gran preponderancia en el contexto internacional en los últimos 40 años. Algunos países de diversos continentes y, especialmente, de América se han posicionado como destinos ecoturísticos. En el marco de los escenarios globalizados, tópicos como los ambientales y el turismo han logrado especial relevancia, ya que a través de estos se puede velar por la conservación de las diferentes especies, incluida la especie humana. El turismo ha consolidado su definición y la ha hecho mucho más trascendental, ya que representa una de las actividades más crecientes, por cuanto aglutina un número cada vez mayor de personas.

Por esta razón es perentoria la implementación y formulación de ciertos insumos de la política, aclaración de conceptos, con el objetivo de poder establecer que tan fructífero pueden ser estos dos sectores, y a través de ellos, como se puede llegar a desarrollar una unidad de negocios mediante la conjunción de estos criterios antes antagónicos.

Rodríguez (2000) establece que nuestro país está considerado como uno de los países más ricos en materia de biodiversidad, considerando a Colombia como uno de los 13 países como mega diversidad, esto sin tener en cuenta la invaluable diversidad cultural. Todo esto nos abre un gran campo de acción para llegar a diversas formas de uso de los recursos, en especial el turismo practicándolo de una forma sostenible y responsable, mediante la especialización que ha traído consigo una creciente demanda de todo lo que está ligado a la naturaleza. En Colombia se ha fortalecido la oferta de servicios turísticos en escenarios naturales, que se han ido apalancando tanto en áreas con protección de carácter nacional, a través de restricciones especiales, en otras áreas protegidas con carácter regional o municipal y en áreas de reserva de sociedad civil bajo el control, inspección y vigilancia de entidades u organizaciones gubernamentales o no gubernamentales. 


\section{MÉTODO}

Como el presente artículo es derivado de una investigación cualitativa la cual es particularmente recomendada para hallar resultados significativos en el comportamiento que las personas conceden a sus experiencias (Hoshmand, 1989; Polkinghorne, 1991), el método seleccionado será la revisión de material existente y referentes en fuentes secundarias, lo que permitirá identificar el entendimiento de los empresarios que serán abordados acerca de sus experiencias culturales (Glaser, 1978), concepción de hipótesis de trabajo o afirmaciones (Erickson, 1986) de los datos y análisis de narrativas de los participantes y sus percepciones. Como instrumento de recolección de información las entrevistas a empresarios del sector turístico en el departamento (Savin-Baden y Major, 2013), el análisis de documentos, registros y artefactos sobre el tema. También se usará la estrategia de Grupos de Enfoque (Morgan 1997 y Barbour, 2007).

El ejercicio obedece a una investigación descriptiva de tipo explicativo y de corte transversal, en donde se buscó caracterizar las condiciones de la población objeto de análisis e identificar si se cumplen las características para que dichas empresas puedan formar parte de un cluster empresarial. Este método incluye cuatro etapas que son descritos en el presente artículo, derivadas de la investigación que le dá origen:

a) La definición de las variables que serán objeto de estudio: En esta etapa se hace la revisión , el planteamiento y la formulación desde el punto de vista teórico, para identificar y definir conceptualmente los ítems que serán observables en desarrollo de la investigación que origina el presente artículo.

b) La diferenciación de las hipótesis y su interacción entre las variables objeto de estudio. En esta etapa se formularon tres hipótesis, que seguramente pueden verse reforzadas o ampliadas en la medida en que el proceso de investigación avance y de cuenta de nuevos hallazgos por parte de los investigadores

c) El establecimiento de los límites a partir de los cuales, los investigadores esperan que las hipótesis se operacionalicen. En esta etapa se tuvo en cuenta el nivel de cumplimiento de las variables contenidas en la investigación, con las características de las empresas para convertirse en miembros de un cluster turístico

d) El análisis de los hallazgos, resultados, escalas y criterios de validez. En esta última etapa se espera analizar los instrumentos de intervención, sus escalas, su validez y su medición, así como la instrumentalización y sistematización de los resultados obtenidos por los investigadores en su intervención, y las características mediante las cuales operan las variables y se pueden generalizar.

\section{Formulación de hipótesis}

Después de analizar los referentes teóricos citados anteriormente, se formulan las siguientes hipótesis: 
H1: El desconocimiento de los clústeres como modelo empresarial puede perjudicar, primeramente, a los empresarios turísticos en el Departamento de Cundinamarca

H2: El desconocimiento de los clústeres como modelo empresarial puede afectar La competitividad en las empresas del sector turístico en el Departamento de Cundinamarca

H3: Los clústeres como modelo empresarial contribuyen al fortalecimiento de las condiciones de negociación de los empresarios del sector turístico en el departamento de Cundinamarca.

\section{RESULTADOS}

Por ser un artículo que forma parte de un proyecto de investigación en su fase inicial, se espera que la investigación refleje de forma concreta, toda la información probable desde diferentes ángulos y perspectivas de los empresarios del sector del turismo, mediante la aplicación de fuentes e instrumentos como las descripciones escritas, las entrevistas, los audios y los videos. Esperamos que la información recopilada, describa ampliamente la percepción que tienen los empresarios respecto de la influencia de los clústeres en los procesos de competitividad empresarial del sector turístico en Cundinamarca, para entender la complejidad de su desconocimiento y las potenciales consecuencias que pueda traer su implementación para los empresarios del sector objeto de estudio.

\section{DISCUSIÓN Y CONCLUSIONES}

\subsection{Discusión}

$\mathrm{Al}$ analizar la zona geográfica en donde se abordará el estudio sobre la viabilidad del clúster a partir del análisis zonal, mediante un Foda, se evidencia en el diagnóstico de la Sabana Occidente, a partir del estudio de las Fortalezas, Debilidades, Oportunidades y Amenazas,y una vez contrastada esta información con actores locales de la jurisdicción. Se destacan los diferentes factores internos y externos determinados como los de mayor relevancia por los protagonistas locales y que permitieron confirmar el origen de un estudio distintivo entre los diferentes fenómenos tanto internos y externos tenidos en cuenta.

Un estudio del $\operatorname{Cepec}^{2}(2011)$ advierte la viabilidad para que la Sabana de Occidente, cumpla con las condiciones para clusterizarse en diferentes sectores a partir de la siguiente información;

Dentro de las fortalezas se destacan entre otras:

- Un importante volumen de productos agrícolas en cosecha permanente durante casi todo el año.

2. Centro de Pensamiento en Estrategias Competitivas-Cepec-Universidad del Rosario 
- El área geográfica objeto de esta propuesta, es una de las de mayor concentración empresarial del departamento (se destacan Mosquera, Facatativá, Funza y Madrid).

- La Sabana de occidente es la segunda zona geográfica del departamento con mayor contribución al PIB departamental, superada solamente por la Sabana centro.

- El sector de la floricultura es el sector que mas contribuye a la generación de empleo en el departamento y de la misma manera, contribuye con una importante cifra de exportaciones regionales.

- El sector industrial es le que más suma en la sumatoria del PIB de la provincia con un $35.4 \%$.

- La Sabana de occidente es la zona geográfica del departamento con mayor contribución de la población en edad de trabajar con formación universitaria, por su cercanía con la capital de la república.

Por otro lado, teniendo en cuenta las debilidades identificadas en el estudio del Cepec, se debe destacar entre otras las siguientes:

- No se puede identificar fácilmente en la región una preferencia en las unidades económicas por ninguno de los sectores y subsectores de la economía, en el marco de un desarrollo regional sostenible.

- En materia de cobertura de servicios públicos, en las áreas rurales el porcentaje aún es bajo, lo que desestimularía una potencial oferta de servicios turísticos en el corto plazo.

- La satisfacción de la demanda de alimentos en otras regiones incrementa los costos en el abastecimiento y distribución de los productos en los municipios que forman parte del área geográfica departamental analizada.

En materia de oportunidades de desarrollo, podemos destacar lo siguiente:

- Una gran oportunidad de crecimiento y desarrollo derivado de la proximidad a la ciudad de Bogotá lo que posibilita su adecuado aprovechamiento.

- Proyectos en desarrollo de Ampliación y Remodelación del Aeropuerto Internacional El Dorado, si se tiene en cuenta por parte de legisladores y directivos involucrados como un proyecto de impacto regional que posibilitaría extender su impacto a la región por las facilidades que traería consigo.

- Alta factibilidad de generación de emprendimientos empresariales de diferente índole, tanto urbanas, como regionales y ambientales, que cumplen con la legislación vigente como consecuencia de una propensión por la reubicación geográfica de industrias y servicios logísticos hacia los espacios regionales (parques industriales, científicos, tecnológicos y logísticos de clase mundial). 
Estudiando las amenazas establecidas en la investigación de la Universidad del Rosario, podemos destacar entre otras las siguientes:

- Preocupación por las dificultades sociales que pueden traducirse en comportamientos delictivos tales como vandalismo, robos, etc; a raíz de la alta concentración de población en algunos municipios de la zona.

- Porcentaje muy importante de población desplazada que se concentra en algunos municipios de la provincia.

- Preocupante crecimiento en las condiciones de inseguridad a partir del aumento de población flotante y que sigue llegando a los municipios.

- Competencia con otras regiones del país.

- Quebrantamiento de la regulación ambiental, que atenta contra los paraísos econturisticos de la zona.

\subsection{Conclusiones}

El estudio de la literatura relacionada muestra lo relevante del establecimiento de tramados empresariales y su vínculo con la aptitud de la organización en la ejecución de tareas en materia de innovación y desarrollo con el fin de lograr progresos en materia de desarrollo de producto, establecimiento de procesos organizados. En línea con esta mirada, la presente propuesta analizó el impacto de las correspondencias entre organizaciones empresariales en la búsqueda de avances, ventajas competitivas, procesos de innovación de la propia organización y su interrelación con las otras empresas ubicadas en el mismo entorno regional y departamental, especialmente en el clúster turístico del departamento de Cundinamarca.

Existe una importante potencialidad para que la Sabana de occidente se convierta en territorio fértil para la generación de clústeres turísticos. La presente afirmación se sustenta con base en dos documentos básicos; (i)el plan de Competitividad que fue estimulado por la Comisión Regional de Competitividad para la Ciudad de Bogotá y el departamento de Cundinamarca ${ }^{3}$ y (ii) la orientación de la Comisión Económica Para América y el Caribe (Cepal) ${ }^{4}$. Como respuesta a la interpretación y análisis de los documentos anteriormente citados, se identificaron cinco puntos fundamentales en los que las autoridades regionales y el sector productivo en general debe centrar sus intenciones de intervención (internacionalización; capital humano y empleo; sostenibilidad ambiental; transformación productiva, innovación y desarrollo tecnológico; y desarrollo de clúster), que de

3. Este plan fue presentado a partir de una percepción de contexto regional, a partir del reconocimiento de seis pilares primordiales; se relacionan a continuación: internacionalización, infraestructura, capital humano e innovación, sostenibilidad ambiental, transformación productiva, y desarrollo de clústeres.

4. Perspectiva que se instrumentaliza a partir del contexto económico de la región, mediante la interpretación del desarrollo competitivo desde una mirada que facilite la inclusión social, y que propone núcleos estratégicos tales como: Infraestructura y localización, Crecimiento, Recursos naturales, Competitividad externa, Capital humano, Calidad de vida, Empresas, Innovación y tecnología, Instituciones, Gestión del gobierno, Inserción en la economía mundial. 
ser intervenidos y explotados tendrían como consecuencia un incremento trascendente de la competitividad y de las características de vida de los habitantes de la Sabana Occidente en el departamento de Cundinamarca.

Tabla No. 1. Definición de Puntos fundamentales de intervención en las provincias del departamento de Cundinamarca

\begin{tabular}{|l|l|}
\hline \multicolumn{1}{|c|}{ ELEMENTOS DE INTERVENCIÓN } & \multicolumn{1}{c|}{ ÁMBITOS DE OPERACIÓN } \\
\hline Internacionalización & $\begin{array}{l}\text { Conquistar la Inversión, fortalecimiento del po- } \\
\text { tencial Exportador y fomento internacional. }\end{array}$ \\
\hline $\begin{array}{l}\text { Recursos Humanos y empleabili- } \\
\text { dad }\end{array}$ & $\begin{array}{l}\text { Adiestramiento y capacitación del capital huma- } \\
\text { no regional. }\end{array}$ \\
\hline Ecointeligencia ambiental & $\begin{array}{l}\text { Estimular los procesos de producción limpia y } \\
\text { eficiente, comercios verdes, seguridad a recursos } \\
\text { naturales, Disposición de Residuos Sólidos. }\end{array}$ \\
\hline $\begin{array}{l}\text { Innovación y desarrollo y estímulo } \\
\text { a la transformación productiva }\end{array}$ & $\begin{array}{l}\text { Estimulo al empresarismo, regularización empre- } \\
\text { sarial, Cierre de brechas tecnológicas, flexibiliza- } \\
\text { ción y facilitación en tramites, estimulo a la inver- } \\
\text { sión en I\&D, recursos de apalancamiento. }\end{array}$ \\
\hline $\begin{array}{l}\text { Incremento y fortalecimiento de } \\
\text { clúster }\end{array}$ & $\begin{array}{l}\text { Cadenas productivas dependiendo de la voca- } \\
\text { ción geográfica de las regiones, asociatividad, } \\
\text { aprendizaje asociativo y cooperativo. }\end{array}$ \\
\hline
\end{tabular}




\section{Referencias}

1. BARBOUR, R. (2007) Doing Focus Group. Londres: Sage. [Trad. Cast.: Grupos de discusión (en prensa). Madrid. Morata. 2011.]

2. BENSON-Rea, M.; WILSON, H. (2003). Networks, learning and the lifecycle. European Management Journal, 21(5), 588-597.

3. BUENDIA, A. (2005). Modelo de Clústeres Industriales. Catarina.udlap.mx/u_dl_a/tales/documentos/mcap/soriano_m_ma/capitulo3.pdf

4. CARBONARA, N. (2002). New models of inter-firm networks within industrial districts. Entrepreneurship \& Regional Development, 14(3), $229-246$.

5. CENTRO DE PENSAMIENTO EN ESTADÍSTICAS COMPETITIVAS - CEPEC (2011). Universidad del Rosario. Bogotá.

6. DWYER, L. \& Kim, C. (2003) “Destination competitiveness: determinants and indicators”. Current issues in Tourism 6(5): 369-414.

7. ERICKSON, F. 1986. Qualitative methods in research on teaching. In Merlin Wittrock, editor, Handbook of research on teaching. Washington, DC.

8. FELDMAN, M.P., J. Francis, and J. Bercovitz. (2005). "Creating a cluster while building a firm: entrepreneurs and the formation of industrial clusters". Regional Studies, 39: 129-141.

9. GALLARZA, I.Et Al (2002). Destination image: towards a conceptual framework Annals of tourism research, 29 (1) (2002), pp. 56-78.

10. GLASER, B. (1978). Theoretical Sensitivity: Advances in the Methodology of Grounded Theory. Mill Valley, Ca.: Sociology Press.

11. HOSHMAND, L. T. (1989). Alternate research paradigms: A review and teaching proposal. The Counseling Psychologist, 17, 3-79.

12. KRUGMAN, P. (1991) Geografía y Comercio Barcelona. Antoni Bosch, editor. 152 pp.

13. LANQUAR, R. (2001), Marketing Turístico. Editorial Ariel.

14. LEIPER, N. (1979) The framework of tourism, Annals of Tourism Research 6(4): 390-407.

15. MARSHALL, A. (1920). Principles of Economics, McMillan, London.

16. MARTín, R. (2006). Principios, organización y práctica del turismo. Tomo 1. Edición Digital (en este mismo CD). Centro de Estudios Turísticos de la Universidad de La Habana.

17. MINGUZZI, A. PASSARO, R. (2000). The network of relationships between the economic environment and the entrepreneurial culture in small firms. Journal of Business Venturing, 16(2), 181-207.

18. MIOSSEC, J.( 1977). “Un modèle de l'espace touristique". L'Espace géographique 6 (1): 41-48.

19. MORGAN, D. (1997). Focus Groups as Qualitative Research. Qualitative Research Methods Series, Volume 16 (second edition). Thousand Oaks, London, New Delhi: Sage Publications.

20. POLKINGHORNE, D. E. (1991). Narrative and self-concept. Journal of Narrative and Life History, 2 \& 3, 135-153.

21. PORTER, M. (1990). The Competitive Advantage Of Nations. Harvard Business Review, pp. 74-91.

22. PORTER, M. (1998). "Clusters and the new economics of competition", Harvard Business Review, noviembre-diciembre.

23. RITCHIE, J, CROUCH, G. (2000). "The competitive destination. A sustainable perspective”. Tourism Management, 21:1-7.

24. RODRíGUEZ, M. (2000). “Ecología y medio ambiente” en Tirado, Álvaro (Ed.). Nueva Historia de Colombia, Vol. IX. Editorial Planeta, Santafé de Bogotá.

25. SAVIN-Baden, M. MAJOR, C. (2013) Qualitative Research: The Essential Guide to Theory and Practice. London: Routledge. Available from http:// www.routledge.com/books/details/9780415674782/

26. STABLER, M. (1988). The image of destination regions: theoretical and empirical aspects, Goodall, B. and Ashworth, G. (Editors), Marketing in the Tourism IndustryĐthe Promotion of Destination Regions. London: Routledge, 133,159.

27. TELISMAN-KOSUTA, N. (1989). Tourist Destination Image. II In Tourism Marketing and Management Handbook, edited by S. F. Witt and L. Moutinho. New York: Prentice Hall, pp. 557-561. 